\title{
Decreasing aversion under ambiguity
}

\author{
Frédéric Cherbonnier \\ Toulouse School of Economics (IEP and IDEI) \\ Christian Gollier ${ }^{1}$ \\ Toulouse School of Economics (LERNA and IDEI)
}

June 3, 2011

${ }^{1}$ This research was supported by the Chair of the Financière de la Cité at TSE, by the Chair SCOR-IDEI, and by the European Research Council under the European Community's Seventh Framework Programme (FP7/2007-2013) Grant Agreement no. 230589. 


\begin{abstract}
Under which condition does the set of desirable uncertain prospects expand when wealth increases? We show that the decreasing concavity (DC) of the utility function $u$ is necessary and sufficient in the $\alpha$-maxmin expected utility model. In the smooth ambiguity aversion model with the ambiguity valuation function $\phi$, the DC of $u$ and of $\phi \circ u$ is necessary and sufficient. An alternative definition of decreasing aversion is based on the hypothesis that the investment in a risky asset is increasing in wealth. We show that this hypothesis does not hold in general under ambiguity aversion, and that one needs to constrain the structure of ambiguity to obtain unambiguous results of an increase in wealth in this portfolio choice problem.
\end{abstract}

Keywords: Decreasing concavity, portfolio choice, $\alpha-\mathrm{MEU}$, smooth ambiguity aversion, maxmin. 


\section{Introduction}

How does one's attitude towards risk evolve when one becomes wealthier? One of the most ubiquitious assumption in the economics of risk is that wealthier people are less risk-averse. Various definitions of the concept of decreasing aversion exist in the literature. For example, an agent is said to have decreasing aversion if any risk that is undesirable at some specific wealth level is also undesirable at all smaller wealth levels. Another definition of decreasing aversion is that in the one-risk-free-one-risky-asset portfolio choice problem, the demand for the risky asset is an increasing function of the initially sure wealth of the agent. In the classical expected utility model, these two definitions of decreasing aversion are equivalent, and the necessary and sufficient condition is expressed by the decreasing nature of the Arrow-Pratt index of absolute risk aversion (DARA). DARA just means that the utility function $u$ exhibits decreasing concavity à la Arrow-Pratt, i.e., that its index of concavity $-u^{\prime \prime}(z) / u^{\prime}(z)$ is decreasing in $z$. This universally accepted property of individual risk preferences plays a crucial role in many applications of the expected utility theory, as illustrated in Gollier (2001).

In this paper, we explore the concept of decreasing aversion in the context of ambiguity and ambiguity aversion. In most cases, the probability distribution of the risk is not perfectly known, i.e., it is ambiguous. Examining a simple thought experiment, Ellsberg (1961) suggested that economic agents do not behave accordingly to the subjective expected utility model. Under ambiguity, contrary to Savage (1954) theory of subjective expected utility, they do not use a subjectively chosen probability distribution to compute the expected utility of the set of possible acts to determine their optimal strategy. Many experiments have confirmed Ellsberg's hypothesis that in the absence of an objective probability distribution, individuals tend to favour a relatively pessimistic plausible distribution to measure their welfare ex ante. Gilboa and Schmeidler (1989) were the first to propose a decision criteria that is compatible with Ellsberg's hypothesis, and that generalizes the expected utility model. In short, agents are assumed to have multiple priors whose formation is a characteristic of the preferences of the agent. The agent's ex ante welfare associated to an act is the smallest expected utility generated by this act over the different possible priors.

More recently, two models have been proposed to account for ambiguity attitude. Ghirardato, Maccheroni and Marinacci (2004) have proposed the 
$\alpha$-maxmin expected utility ( $\alpha$-MEU) family of preferences in which the agent's ex ante welfare is measured by a $\alpha$-weighted average of the smallest and the largest expected utility levels among a convex, compact set of probability distributions. The alternative approach provided by Klibanoff, Marinacci and Mukerji (2005) represents the agent's welfare under uncertainty by the certainty equivalent of the different prior-dependent expected utility levels. This certainty equivalent is computed by using a function $\phi$ that is increasing and concave, and whose degree of concavity is an index of ambiguity aversion.

For these two decision criteria under ambiguity, we determine the conditions under which wealthier people are less averse to risk, under the two standard definitions for this concept. Consider first the definition of decreasing aversion based on the shrinkage of the set of desirable lotteries when wealth is reduced. In the $\alpha-$ MEU family of preferences, this property is obtained under the necessary and sufficient condition that the utility function exhibits DARA. In the case of smooth ambiguity aversion, the shrinkage of the set of desirable lotteries when wealth decreases prevails if and only if both $u$ and $\phi \circ u$ exhibit decreasing concavity à la Arrow-Pratt. This condition is weaker than the sufficient condition that $u$ and $\phi$ are decreasingly concave.

The definition of decreasing aversion based on the increasing demand for the risky asset when wealth increases is more complex to characterize. In the maxmin model, which is a special case of the $\alpha-\mathrm{MEU}$ criterion, we show that the decreasing concavity of the utility function is not enough to guarantee the desired comparative statics property, except in the small. Different sufficient conditions are derived. For example, a sufficient condition is that the utility function belongs to the HARA class with decreasing aversion. Another sufficient condition is that all priors can be ranked according to the Jewitt's order, and relative prudence is smaller than relative risk aversion plus one. A similar condition is obtained in the KMM's smooth ambiguity aversion model, under the addictional condition that $\phi$ is decreasingly concave. The condition relating relative prudence and relative risk aversion may be removed at the cost of replacing the Jewitt's order by the monotone likelihood ratio order, which is stronger. 


\section{Acceptance of risk}

In this section, we characterize the conditions under which wealthier people have a larger set of desirable loteries.

Definition 1 We say that an agent is Decreasingly Averse if a reduction in wealth can never makes an undesirable uncertain situation desirable.

In other words, the set of undesirable lotteries shrinks when wealth increases. In that case, we say that the agent exhibits Decreasing Aversion (DA). The benchmark model is expected utility. Under this model, decreasing aversion is characterized by the following condition. For any initial wealth $z$, and for any random variable $\widetilde{x}$ so that the support of $z+\widetilde{x}$ is in the domain of the utility function $u$, we have that

$$
E u(z+\widetilde{x}) \leq u(z) \Longrightarrow E u\left(z^{\prime}+\widetilde{x}\right) \leq u\left(z^{\prime}\right) \quad \forall z^{\prime} \leq z .
$$

This means that if an agent with utility $u$ dislikes lottery $\widetilde{x}$ at wealth level $z$, she must also dislike it at all wealth levels smaller than $z$. We know since Pratt (1964) that this is true if and only if the utility function $u$ exhibits decreasing absolute risk aversion (DARA). We formalize this by using the following definition.

Definition 2 We say that a function $f: \mathbb{R} \rightarrow \mathbb{R}$ satisfies (weak) Decreasing Concavity $(D C)$ if $-f^{\prime \prime} / f^{\prime}$ is non-increasing.

It is easy to check that $f$ DC means that there exists a concave function $g$ such that $-f^{\prime}=g \circ f$. In the expected utility model, decreasing aversion holds if and only if $u$ exhibits DC. Notice that condition (1) is equivalent to the single-crossing from below of function $v$ defined as $v(z)=E u(z+\widetilde{x})$ with respect to function $u$. Thus, condition (1) is equivalent to

$$
E u(z+\widetilde{x})=u(z) \Longrightarrow E u^{\prime}(z+\widetilde{x}) \geq u^{\prime}(z)
$$

Suppose now that there is some ambiguity about the true distribution of the payoff of the lottery. For the sake of simplicity, suppose that the payoff of the lottery has $n$ possible distributions, corresponding to random variables

$\widetilde{x}_{1}, \ldots, \widetilde{x}_{n}$. Departing from the EU model, we hereafter examine two decision 
models under uncertainty: $\alpha-\mathrm{MEU}$ and smooth ambiguity aversion. Under the $\alpha$-MEU model with $\alpha \in[0,1]$, the agent measures his/her welfare under uncertainty by a $\alpha$-weighted average of the smallest and the largest expected utility levels. In this context, decreasing aversion requires for any $z$, and for any set of random variables $\left(\widetilde{x}_{1}, \ldots, \widetilde{x}_{n}\right)$ so that the support of $z+\widetilde{x}_{\theta}$ is in the domain of the utility function $u$ for all $\theta=1, \ldots, n$,

$$
\alpha \min _{\theta} E u\left(z+\widetilde{x}_{\theta}\right)+(1-\alpha) \max _{\theta} E u\left(z+\widetilde{x}_{\theta}\right) \leq u(z)
$$

implies that $\forall z^{\prime} \leq z$

$$
\alpha \min _{\theta} E u\left(z^{\prime}+\widetilde{x}_{\theta}\right)+(1-\alpha) \max _{\theta} E u\left(z^{\prime}+\widetilde{x}_{\theta}\right) \leq u\left(z^{\prime}\right) .
$$

This means that if the safe prospect is preferred to the uncertain one when initial wealth is $z$, this is also true for all wealth levels smaller than $z$.

Proposition 1 The $\alpha-M E U$ criterion implies decreasing aversion if and only if $u$ exhibits decreasing concavity.

Proof: Suppose by contradiction that there exists $z$ and $z^{\prime} \leq z$ such that

$$
\alpha \min _{\theta} E u\left(z+\widetilde{x}_{\theta}\right)+(1-\alpha) \max _{\theta} E u\left(z+\widetilde{x}_{\theta}\right) \leq u(z)
$$

and

$$
\alpha \min _{\theta} E u\left(z^{\prime}+\widetilde{x}_{\theta}\right)+(1-\alpha) \max _{\theta} E u\left(z^{\prime}+\widetilde{x}_{\theta}\right)>u\left(z^{\prime}\right) .
$$

Let us define

$$
a=\arg \min _{\theta} E u\left(z+\widetilde{x}_{\theta}\right)
$$

and

$$
b=\arg \max _{\theta} E u\left(z^{\prime}+\widetilde{x}_{\theta}\right) .
$$

Because $\alpha \in[0,1]$, these definitions imply that

$\alpha E u\left(z+\widetilde{x}_{a}\right)+(1-\alpha) E u\left(z+\widetilde{x}_{b}\right) \leq \alpha \min _{\theta} E u\left(z+\widetilde{x}_{\theta}\right)+(1-\alpha) \max _{\theta} E u\left(z+\widetilde{x}_{\theta}\right)$

and

$\alpha E u\left(z^{\prime}+\widetilde{x}_{a}\right)+(1-\alpha) E u\left(z^{\prime}+\widetilde{x}_{b}\right) \geq \alpha \min _{\theta} E u\left(z^{\prime}+\widetilde{x}_{\theta}\right)+(1-\alpha) \max _{\theta} E u\left(z^{\prime}+\widetilde{x}_{\theta}\right)$ 
Let us define the random variable $X$ by its distribution $\left(\alpha, \widetilde{x}_{a} ; 1-\alpha, \widetilde{x}_{b}\right)$. Combining inequalities (5) and (6) implies that $E u(z+X) \leq u(z)$. Similarly, combining inequalities (7) and (8) implies that $E u\left(z^{\prime}+X\right)>u\left(z^{\prime}\right)$. In short, a reduction in wealth from $z$ to $z^{\prime}$ makes the initially undesirable lottery $X$ desirable. This is a contradiction.

We can conclude from this result that the multi-prior $\alpha-\mathrm{MEU}$ model has the property of decreasing aversion if and if the corresponding expected utility model has it also, which is the case if $-u^{\prime \prime} / u^{\prime}$ is non-increasing. This result applies in particular in the special cases of the maxmin and the maxmax models.

We now examine the model of smooth ambiguity aversion, as introduced by Klibanoff, Marinacci and Mukerji (KMM, 2005). This KMM model is based on the same ingredients than the maxmin model. A new ingredient is the vector $\left(q_{1}, \ldots, q_{n}\right)$ of non-negative scalars that sum up to unity. Parameter $q_{\theta}$ can be interpreted as the (subjective) probability that $\widetilde{x}_{\theta}$ describes the true distribution of the lottery. Another ingredient is a new real-valued function $\phi$ that is increasing and concave, so that the welfare if the lottery is accepted is measured by the certainty equivalent of the conditional expected utility $E u\left(z+\widetilde{x}_{\theta}\right)$ :

$$
W(z)=\phi^{-1}\left(\sum_{\theta=1}^{n} q_{\theta} \phi\left(E u\left(z+\widetilde{x}_{\theta}\right)\right)\right)
$$

Because $\phi$ is assumed to be concave, the existence of ambiguity reduces welfare since, by Jensen's inequality,

$$
W(z) \leq \phi^{-1}\left(\phi\left(\sum_{\theta=1}^{n} q_{\theta} E u\left(z+\widetilde{x}_{\theta}\right)\right)\right)=E u(z+\widetilde{x}),
$$

where $\widetilde{x}$ is distributed as $\left(q_{1}, \widetilde{x}_{1} ; \ldots ; q_{n}, \widetilde{x}_{n}\right)$. If the lottery is rejected, the agent enjoys welfare $\phi^{-1}(\phi(u(z)))=u(z)$. Decreasing aversion as defined in this section requires in this case that $W$ single-crosses $u$ from below. This requires that the following property be satisfied:

$\phi^{-1}\left(\sum_{\theta=1}^{n} q_{\theta} \phi\left(E u\left(z+\widetilde{x}_{\theta}\right)\right)\right)=u(z) \Longrightarrow \frac{\sum_{\theta=1}^{n} q_{\theta} \phi^{\prime}\left(E u\left(z+\widetilde{x}_{\theta}\right)\right) E u^{\prime}\left(z+\widetilde{x}_{\theta}\right)}{\phi^{\prime}(W(z))} \geq u^{\prime}(z)$ 
One can easily extract two necessary conditions for decreasing aversion in this framework. The first is that $u$ must be DC, since one possibility is that all $\widetilde{x}_{\theta}$ be identically distributed. Indeed, in that case, condition (9) is equivalent to (2). The second condition can be derived in the special case in which all $\widetilde{x}_{\theta}$ are degenerated. Suppose that $\widetilde{x}_{\theta}$ takes value $y_{\theta}$ almost surely, for all $\theta=1, \ldots, n$. Let random variable $\widetilde{y}$ be distributed as $\left(y_{1}, q_{1} ; \ldots ; y_{n}, q_{n}\right)$. Then, condition (9) can be rewritten as

$$
E \phi \circ u(z+\widetilde{y})=\phi \circ u(z) \Longrightarrow E(\phi \circ u)^{\prime}(z+\widetilde{y}) \geq(\phi \circ u)^{\prime}(z) .
$$

As recalled above, this condition holds for all $z$ and all random variable $\widetilde{y}$ if and only if $\phi \circ u$ exhibits DC. This observation is just a restatement of the fact that the KMM model simplifies to the expected utility model with utility function $\phi \circ u$ when the multiple priors are all degenerated.

This means that both conditions $u \mathrm{DC}$ and $\phi \circ u \mathrm{DC}$ are necessary for decreasing aversion in the KMM smooth ambiguity model. We hereafter show that this joint condition is also sufficient.

Proposition 2 Consider the KMM smooth ambiguity aversion model characterized by functions $(u, \phi)$. It exhibits decreasing aversion if and only if $u$ and $\phi \circ u$ both exhibit decreasing concavity.

Proof of sufficiency: Let $y_{\theta}$ be the certainty equivalent of $\widetilde{x}_{\theta}$ under function $u$, i.e., $u\left(z+y_{\theta}\right)=E u\left(z+\widetilde{x}_{\theta}\right)$. Let random variable $\widetilde{y}$ be distributed as $\left(y_{1}, q_{1} ; \ldots ; y_{n}, q_{n}\right)$. Suppose that

$$
W(z)=\phi^{-1}\left(\sum_{\theta=1}^{n} q_{\theta} \phi\left(E u\left(z+\widetilde{x}_{\theta}\right)\right)\right)=u(z),
$$

or equivalently, that $E \phi \circ u(z+\widetilde{y})=\phi \circ u(z)$. Because $u$ is DC, $E u\left(z+\widetilde{x}_{\theta}\right)=$ $u\left(z+y_{\theta}\right)$ implies that $E u^{\prime}\left(z+\widetilde{x}_{\theta}\right) \geq u^{\prime}\left(z+y_{\theta}\right)$. It implies in turn that

$$
\sum_{\theta=1}^{n} q_{\theta} \phi^{\prime}\left(E u\left(z+\widetilde{x}_{\theta}\right)\right) E u^{\prime}\left(z+\widetilde{x}_{\theta}\right) \geq \sum_{\theta=1}^{n} q_{\theta} \phi^{\prime}\left(u\left(z+y_{\theta}\right)\right) u^{\prime}\left(z+y_{\theta}\right)=E(\phi \circ u)^{\prime}(z+\widetilde{y}) \text {. }
$$

We assume that $\phi \circ u$ is DC. It implies that $E(\phi \circ u)^{\prime}(z+\widetilde{y}) \geq(\phi \circ u)^{\prime}(z)$. We conclude from the previous equation that

$$
\sum_{\theta=1}^{n} q_{\theta} \phi^{\prime}\left(E u\left(z+\widetilde{x}_{\theta}\right)\right) E u^{\prime}\left(z+\widetilde{x}_{\theta}\right) \geq \phi^{\prime}(u(z)) u^{\prime}(z)=\phi^{\prime}(W(z)) u^{\prime}(z) .
$$


It implies that the right condition in (9) is satisfied. This concludes the proof of the sufficiency of $u$ and $\phi \circ u$ being DC.

An interesting case arises when $u$ exhibits constant absolute risk aversion and $\phi$ is a power function:

$$
u(c)=-\frac{e^{-A c}}{A} \in \mathbb{R}_{-} \quad \text { and } \quad \phi(\approx)=-\frac{(-\widetilde{\simeq})^{\nVdash+\gamma}}{\nVdash+\gamma},
$$

with $\gamma \geq 0$. Function $\phi$ is increasing and concave in the relevant domain of $u$. Observe that $-\phi^{\prime \prime}(u) / \phi^{\prime}(u)=-\gamma / u$, which is increasing in $u$. Thus, we have that $u$ and $\phi$ exhibit respectively constant concavity and increasing concavity. However, preferences (10) satisfy the necessary and sufficient condition of Proposition 2, since

$$
(\phi \circ u)(c)=-\frac{e^{-A(1+\gamma) c}}{(1+\gamma) A^{1+\gamma}},
$$

is weakly DC. This shows that this necessary and sufficient condition does not require that $\phi$ exhibits DC. Only the DC of $u$ and $\phi \circ u$ is required. A sufficient condition is that $u$ and $\phi$ be DC. Indeed, we have that

$$
-\frac{(\phi \circ u)^{\prime \prime}(z)}{(\phi \circ u)^{\prime}(z)}=-\frac{\phi^{\prime \prime}(u(z))}{\phi^{\prime}(u(z))} u^{\prime}(z)-\frac{u^{\prime \prime}(z)}{u^{\prime}(z)} .
$$

Because $u$ is increasing and $u^{\prime}$ is decreasing, the right-hand side of this equality is decreasing in $z$ if both $-\phi^{\prime \prime} / \phi^{\prime}$ and $-u^{\prime \prime} / u^{\prime}$ are decreasing. This yields the following corollary.

Corollary 1 Consider the KMM smooth ambiguity aversion model characterized by functions $(u, \phi)$. It exhibits decreasing aversion if both $u$ and $\phi$ exhibit decreasing concavity.

The fact that the DC of $\phi$ is not necessary can be illustrated by the following counterexample. Suppose that $u(z)=z^{1 / 2}$. Suppose also that $\phi$ is such that $\phi^{\prime}(u)=\exp \left(-k u^{2}\right)$, so that $-\phi^{\prime \prime}(u) / \phi^{\prime}(u)=2 k u$. Thus, this $\phi$ function, which is increasing and concave in the relevant domain, exhibits increasing concavity. Still, $\phi \circ u$ exhibits DC, since using (11), we have that

$$
-\frac{(\phi \circ u)^{\prime \prime}(z)}{(\phi \circ u)^{\prime}(z)}=\frac{2 k z^{1 / 2}}{2 z^{1 / 2}}+\frac{1}{2 z}=k+\frac{1}{2 z}
$$

which is decreasing. Thus, from Proposition 2, the smooth ambiguity-averse agent $(u, \phi)$ is decreasingly averse in spite of the fact that $\phi$ is not DC. 


\section{Portfolio allocation}

In this section, we examine an alternative decision model. The decision maker with initial wealth $z$ can invest in two assets. There is a risk free asset whose return is normalized to zero, and a risky asset whose return is expressed by random variable $\widetilde{x}$. The agent must choose the size $\alpha$ of her investment in the risky asset. In the expected utility model, this decision problem can be written as

$$
\max _{\alpha} E u(z+\alpha \widetilde{x}) .
$$

In this model, it is easy to check that an increase in the initial wealth raises the dollar investment in the risky asset if and only if $u$ is decreasingly concave, as first shown by Arrow (1963). Indeed, this requires to show that the cross derivative of the objective function in (12) with respect to $\alpha$ and $z$ evaluated at the optimal $\alpha$ is positive. We normalize the unit of the risky asset in such a way that it is optimal to invest exactly one monetary unit in the risky asset, so that the first order condition of the above program yields

$$
E \tilde{x} u^{\prime}(z+\tilde{x})=0 .
$$

Because $u$ DC means that $-u^{\prime}$ is a concave function $g$ of $u$, it implies that

$$
E \widetilde{x} u^{\prime \prime}(z+\widetilde{x})=-E \widetilde{x} g^{\prime}(u(z+\widetilde{x})) u^{\prime}(z+\widetilde{x}) \geq-g^{\prime}(u(z)) E \widetilde{x} u^{\prime}(z+\widetilde{x})=0 .
$$

The inequality above comes from the observation that for all $x,-x g^{\prime}(u(z+$ $x)) \geq-x g^{\prime}(u(z))$. This demonstrates that the DC property in the expected utility model is necessary and sufficient for two testable results. Namely, the DC of $u$ means that when wealth increases, the set of acceptable lotteries inflates, and the demand for the risky asset increases. In the following, we examine whether this property is still satisfied under ambiguity aversion. As in the previous section, we examine two models of ambiguity aversion: the $\alpha-$ MEU model and the smooth ambiguity aversion model. For expository reasons, we limit the analysis of the $\alpha$-MEU model to its special case of the maxmin criterion. An introduction to the polar case of the maxmax criterion is presented in the Appendix.

\subsection{The maxmin model}

Suppose that the distribution of the return of the risky asset is ambiguous. This ambiguity is characterized by $n$ possible random variables $\left(\widetilde{x}_{1}, \ldots, \widetilde{x}_{n}\right)$. 
Under the maxmin criterion, the decision program can be rewritten as

$$
\max _{\alpha} \min _{\theta} E u\left(z+\alpha \widetilde{x}_{\theta}\right) .
$$

Of course, if there exists a $\theta$ such that $\widetilde{x}_{\theta}$ is dominated by all other $\widetilde{x}_{\theta^{\prime}}, \theta^{\prime} \neq$ $\theta$, then the minimum is obtained with this $\theta$, and the above maximization program is as in the EU model. The next Lemma provides us with a general condition under which an increase of wealth raises the investment in the risky asset. This condition states that whenever the agent with utility $u$ is indifferent between $\widetilde{x}_{i}$ and $\widetilde{x}_{j}$, but would prefer to invest more than one unity in $\widetilde{x}_{i}$ and less than one unity in $\widetilde{x}_{j}$, then the expected marginal utility under $\widetilde{x}_{i}$ is smaller than under $\widetilde{x}_{j}$.

Lemma 1 Suppose that $u$ is DC. In the maxmin model of ambiguity aversion, an increase in wealth increases the demand for the risky asset if, for all $(i, j) \in\{1, \ldots, n\}^{2}$,

$$
\left.\begin{array}{c}
E u\left(z+\tilde{x}_{i}\right)=E u\left(z+\tilde{x}_{j}\right) \\
E \tilde{x}_{i} u^{\prime}\left(z+\tilde{x}_{i}\right) \geq 0 \geq E \tilde{x}_{j} u^{\prime}\left(z+\tilde{x}_{j}\right)
\end{array}\right\} \Longrightarrow E\left[u^{\prime}\left(z+\tilde{x}_{i}\right)\right] \leq E\left[u^{\prime}\left(z+\tilde{x}_{j}\right)\right] .
$$

Proof: Let's consider the optimal investment $\alpha^{*}$ that solves the maximization problem 13. If the minimum $\min _{\theta} E u\left(z+\alpha^{*} \widetilde{x}_{\theta}\right)$ is reached at only one prior $\theta=i \in\{1, \ldots, n\}$, then locally around $z$, the decision program can be rewritten as $\max _{\alpha} E u\left(z+\alpha \widetilde{x}_{i}\right)$ and the decreasing aversion property is a consequence of $u \mathrm{DC}$. Hence, in order to check the decreasing aversion property, we only need to consider cases where there exist $(i, j) \in\{1, \ldots, n\}^{2}$ such that the optimum of the decision problem is such that

$$
E u\left(z+\alpha^{*} \widetilde{x}_{i}\right)-E u\left(z+\alpha^{*} \widetilde{x}_{j}\right)=0,
$$

where $\alpha^{*}$ is normalized to unity. An optimality condition for $\alpha^{*}=1$ at this kink of the objective function is that $E \widetilde{x}_{i} u^{\prime}\left(z+\widetilde{x}_{i}\right)$ and $E \widetilde{x}_{j} u^{\prime}\left(z+\widetilde{x}_{j}\right)$ cannot be both either strictly positive or strictly negative. Without loss of generality suppose that

$$
E \tilde{x}_{i} u^{\prime}\left(z+\tilde{x}_{i}\right) \geq 0 \geq E \tilde{x}_{j} u^{\prime}\left(z+\tilde{x}_{j}\right) .
$$


Now, observe that condition (15) characterizes $\alpha^{*}$ locally around $z$. Fully differentiating this equality with respect to $z$ yields

$$
\frac{d \alpha^{*}}{d z}=-\frac{E u^{\prime}\left(z+\widetilde{x}_{i}\right)-E u^{\prime}\left(z+\widetilde{x}_{j}\right)}{E \widetilde{x}_{i} u^{\prime}\left(z+\alpha^{*} \widetilde{x}_{i}\right)-E \widetilde{x}_{j} u^{\prime}\left(z+\alpha^{*} \widetilde{x}_{j}\right)} .
$$

This is positive if $E\left[u^{\prime}\left(z+\tilde{x}_{i}\right)\right] \leq E\left[u^{\prime}\left(z+\tilde{x}_{j}\right)\right]$.

In fact, sufficient condition (14) is also necessary if $n=2$, or if the set of priors can be modified so that only priors $i$ and $j$ drive the solution, i.e., if the minimal expected utility is a corner solution with $E u\left(z+\alpha^{*} \widetilde{x}_{i}\right)=E u(z+$ $\left.\alpha^{*} \widetilde{x}_{j}\right)$. In that case, as shown in the proof, a reversal in the right inequality in (14) would imply that $\alpha^{*}$ would be locally decreasing in wealth. In Figure 1, we have illustrated condition (14). At wealth level $z$, the objective function $h(a)=\min \left(E u\left(z+\alpha \widetilde{x}_{i}\right), E u\left(z+\alpha \widetilde{x}_{j}\right)\right)$ has a maximum at the kink that is characterized by the two left conditions in (14). We also see on the picture that the right condition in (14) is satisfied. Indeed, the increase in wealth has a larger effect on $E u\left(z+\alpha^{*} \widetilde{x}_{j}\right)$ than on $E u\left(z+\alpha^{*} \widetilde{x}_{i}\right)$. It implies that $\alpha^{*}(z)>\alpha^{*}\left(z^{\prime}\right)$.

Condition (14) is linked to the DC property of $u$. Indeed, suppose that one of the two priors is degenerated at zero. ${ }^{1}$ Suppose for example that $\widetilde{x}_{i}=0$ almost surely. In that special case, condition (14) can be rewritten as follows:

$$
\left.\begin{array}{l}
E u\left(z+\tilde{x}_{j}\right)=u(z) \\
E \tilde{x}_{j} u^{\prime}\left(z+\tilde{x}_{j}\right) \leq 0
\end{array}\right\} \Longrightarrow E\left[u^{\prime}\left(z+\tilde{x}_{j}\right)\right] \geq u^{\prime}(z)
$$

Observe first that $E u\left(z+\tilde{x}_{j}\right)=u(z)$ implies that $E \tilde{x}_{j} u^{\prime}\left(z+\tilde{x}_{j}\right) \leq 0$. Indeed, function $H(\alpha)=E u\left(z+\alpha \widetilde{x}_{j}\right)$ is concave in $\alpha$. Thus, if $H(1)=H(0)$, it must imply that $H^{\prime}(1) \leq 0$. This means that the above condition can be rewritten as

$$
E u\left(z+\tilde{x}_{j}\right)=u(z) \Longrightarrow E\left[u^{\prime}\left(z+\tilde{x}_{j}\right)\right] \geq u^{\prime}(z),
$$

which is the standard DC condition. We can conclude from this observation that if one of the two priors is degenerated, $u$ DC is sufficient to guarantee that, in the maxmin model, wealthier people invest more in the risky asset.

\footnotetext{
${ }^{1}$ It is easy to check that the same result holds when one of the two priors is degenerated elsewhere than at zero.
} 


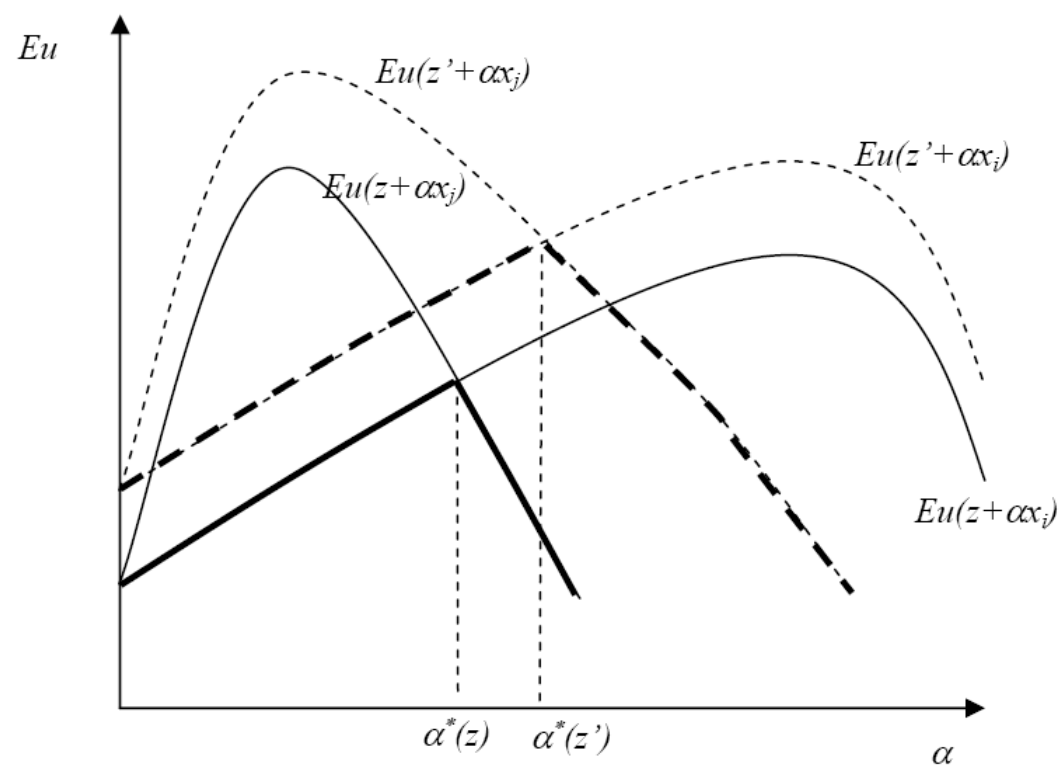

Figure 1: Effect of an increase in wealth from $z$ to $z^{\prime}>z$ on the optimal portfolio allocation in the maxmin model. 
Another way to see the link between DC and condition (14) is to examine the case in which both $\widetilde{x}_{i}$ and $\widetilde{x}_{j}$ are small. In the small, the indifference between $\widetilde{x}_{i}$ and $\widetilde{x}_{j}$ is equivalent to condition

$$
m_{1 i}-0.5 A m_{2 i}=m_{1 j}-0.5 A m_{2 j},
$$

where $m_{k i}$ is the $k$-th moment of random variable $\widetilde{x}_{i}$, and $A=-u^{\prime \prime}(z) / u^{\prime}(z)$ is absolute risk aversion. The condition that the demands for $\widetilde{x}_{i}$ and for $\widetilde{x}_{j}$ are respectively larger and smaller than unity is equivalent to the following condition:

$$
m_{1 i}-A m_{2 i} \geq 0 \geq m_{1 j}-A m_{2 j} .
$$

Replacing $m_{1 i}$ by its expression derived from (17) in the first inequality allows us to rewrite these two inequalities as follows:

$$
A \frac{m_{2 i}+m_{2 j}}{2} \leq m_{1 j} \leq A m_{2 j} .
$$

These two inequalities imply in particular that $m_{2 i}$ be smaller than $m_{2 j} .{ }^{2}$ Intuitively, if two small risks $i$ and $j$ yield the same expected utility, but the demand for $i$ is larger than the demand for $j$, it must be that the riskiness of $i$ is smaller than the riskiness of $j$ (and the expected payoff of $i$ is smaller than the expected payoff of $j$ ).

Now, observe that in the small, condition $E\left[u^{\prime}\left(z+\tilde{x}_{i}\right)\right] \leq E\left[u^{\prime}\left(z+\tilde{x}_{j}\right)\right]$ is equivalent to

$$
m_{1 i}-0.5 P m_{2 i} \geq m_{1 j}-0.5 P m_{2 j},
$$

where $P=-u^{\prime \prime \prime}(z) / u^{\prime \prime}(z)$ is absolute prudence. Using condition (17), this inequality holds if and only if

$$
\left(m_{2 i}-m_{2 j}\right)(A-P) \geq 0 .
$$

Because $m_{2 i} \leq m_{2 j}$ from (19), this condition holds if and only if $P \geq A$, i.e., if and only if $u$ is DC.

To sum up what we have at this stage in the maxmin model, $u$ DC is sufficient for the demand for the risky asset to raises with wealth when one of the two priors is degenerated, or when the different possible priors entails small risk. However, DC is generally not sufficient, as shown in the following counter-example.

\footnotetext{
${ }^{2}$ Notice that the same result holds when condition $E \tilde{x}_{i} u^{\prime}\left(z+\tilde{x}_{i}\right) \geq 0 \geq E \tilde{x}_{j} u^{\prime}\left(z+\tilde{x}_{j}\right)$ is relaxed to $E \tilde{x}_{i} u^{\prime}\left(z+\tilde{x}_{i}\right) \geq E \tilde{x}_{j} u^{\prime}\left(z+\tilde{x}_{j}\right)$.
} 


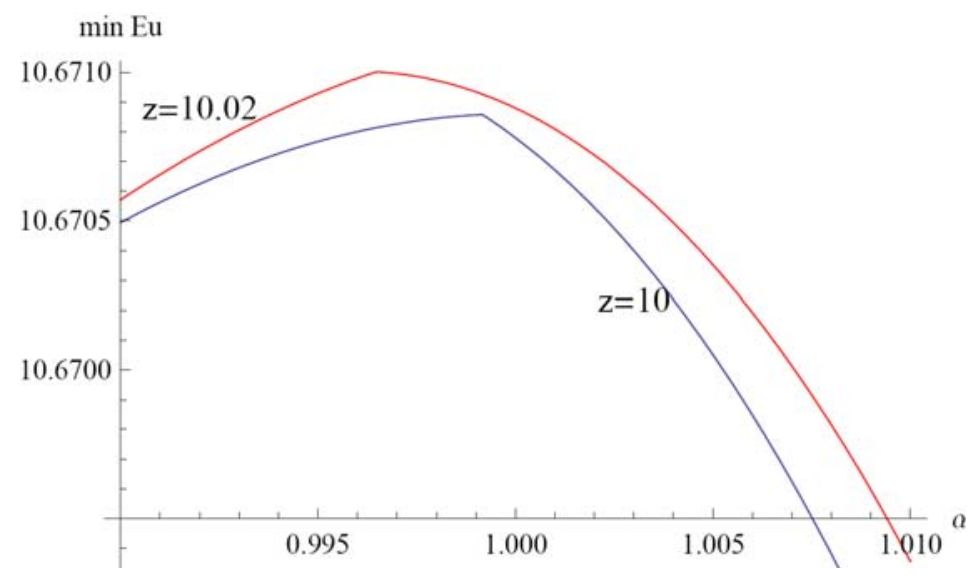

Figure 2: Maxmin welfare as a function of the investment in the risky asset $\alpha$ for three different wealth levels $z$,in the case described in Example 1.The three curves have been translated vertically for the sake of comparison.

Example 1 Consider function $u(c)=c-1 / c$ in $\mathbb{R}^{+}$. This function is increasing, concave and DC. Consider $\widetilde{x}_{1} \sim(-8,0.47437 ; 9,0.52563)$ and $\widetilde{x}_{2} \sim(-9,0.10719 ; 2,0.86155 ; 3,0.03126)$. One can check that $\widetilde{x}_{1}$ and $\widetilde{x}_{2}$ violate condition (14) at $z=10$. In Figure 2, we show that an increase in wealth around $z=10$ reduces the demand for the risky asset.

Definition 3 We say that $\widetilde{x}_{i}$ dominates $\widetilde{x}_{j}$ in the sense of Jewitt if the following condition holds: for all increasing and concave $u$, if agent $u$ weakly prefers $\widetilde{x}_{i}$ to $\widetilde{x}_{j}$, then all agents more risk-averse than $u$ also weakly prefer $\widetilde{x}_{i}$ to $\widetilde{x}_{j}$.

This is denoted $\widetilde{x}_{i} \succeq_{J} \widetilde{x}_{j}$. By definition, if $\widetilde{x}_{i}$ dominates $\widetilde{x}_{j}$ in the sense of Jewitt, $\widetilde{x}_{i}$ also dominates $\widetilde{x}_{j}$ in the sense of the second-order stochastic dominance (SSD). Thus, Jewitt's order is weaker than SSD. Jewitt (1989) fully characterizes this stochastic order. A sufficient condition is that the cumulative distribution function of $\widetilde{x}_{i}$ crosses the cumulative distribution function of $\widetilde{x}_{j}$ only once, from below. Now suppose that we can ranked 
the set of priors $\left\{\widetilde{x}_{1}, \ldots, \widetilde{x}_{n}\right\}$ according to Jewitt's order. Without loss of generality, suppose that $\widetilde{x}_{1} \succeq_{J} \widetilde{x}_{2} \succeq_{J} \ldots \succeq_{J} \widetilde{x}_{n}$. Define function $\psi$ such that $\psi(x)=x u^{\prime}(z+x)$ for all $x$. Suppose that both $-u^{\prime}$ and $\psi$ are more concave than $u$ in the sense of Arrow-Pratt. Consequently, for any $(i, j) \in$ $\{1, \ldots, n\}^{2}$, if $E u\left(z+\widetilde{x}_{i}\right)=E u\left(z+\widetilde{x}_{j}\right)$, then $E u^{\prime}\left(z+\widetilde{x}_{i}\right) \leq E u^{\prime}\left(z+\widetilde{x}_{j}\right)$ and $E \widetilde{x}_{i} u^{\prime}\left(z+\widetilde{x}_{i}\right) \geq E \widetilde{x}_{j} u^{\prime}\left(z+\widetilde{x}_{j}\right)$. This is stronger than requested by condition (14). The conditions in the following proposition guarantee that $-u^{\prime}$ and $\psi$ are more concave than $u$.

Proposition 3 Normalize the demand for the risky asset at wealth level $z$ to unity. Suppose that the set of priors $\left\{\widetilde{x}_{1}, \ldots, \widetilde{x}_{n}\right\}$ can be ranked according to Jewitt's order. In the maxmin model of ambiguity aversion, an increase in wealth around $z$ raises the demand for the risky asset if $u$ is DC and for all $x$ in the joint support of $\left\{\widetilde{x}_{1}, \ldots, \widetilde{x}_{n}\right\}$, we have that

$$
x[P(z+x)-A(z+x)] \leq 1,
$$

where $A$ and $P$ are the indices of absolute risk aversion and of absolute prudence, respectively.

Proof: It remains to prove that $-u^{\prime}$ and $\psi$ are two concave transformations of $u$. We already know that $u$ DC just means that $-u^{\prime}$ is more concave than $u$. Concerning $\psi$, let us define function $k$ such that $\psi(x)=k(u(x))$ in the joint support of $\left\{\widetilde{x}_{1}, \ldots, \widetilde{x}_{n}\right\}$. By definition of $\psi$, fully differentiating this equality twice yields

$$
u^{\prime}(z+x)+x u^{\prime \prime}(z+x)=k^{\prime}(u(z+x)) u^{\prime}(z+x),
$$

and

$2 u^{\prime \prime}(z+x)+x u^{\prime \prime \prime}(z+x)=k^{\prime \prime}(u(z+x))\left(u^{\prime}(z+x)\right)^{2}+k^{\prime}(u(z+x)) u^{\prime \prime}(z+x)$.

Eliminating $k^{\prime}$ from these two equations allows us to write

$$
k^{\prime \prime}(u(z+x))\left(u^{\prime}(z+x)\right)^{2}=u^{\prime \prime}(z+x)-x\left(u^{\prime \prime \prime}(z+x)-\frac{\left(u^{\prime \prime}(z+x)\right)^{2}}{u^{\prime}(z+x)}\right) .
$$

This implies that $k$ is concave in the relevant domain of $x$ if condition (21) is satisfied. 
Observe that this proposition implies that $u \mathrm{DC}$ is sufficient if the possible priors are small risk, that is, if the joint support of priors is in a small neighborhood of 0 . Observe also that condition (21) is quite general, as we show now. Define relative risk aversion and relative prudence respectively as $A^{r}(c)=-c u^{\prime \prime}(c) / u^{\prime}(c)$ and $P^{r}(c)=-c u^{\prime \prime \prime}(c) / u^{\prime \prime}(c)$. Condition (21) can then rewritten as

$$
P^{r}(z+x)-A^{r}(z+x)-z[P(z+x)-A(z+x)] \leq 1 .
$$

Assuming that the domain of possible wealth levels is in $\mathbb{R}^{+}$, a sufficient condition for this inequality is that $P^{r}(z+x)$ is weakly larger than $A^{r}(z+$ $x)+1$ for all $z+x$.

Corollary 2 Normalize the demand for the risky asset at wealth level $z$ to unity. Suppose that the set of priors $\left\{\widetilde{x}_{1}, \ldots, \widetilde{x}_{n}\right\}$ can be ranked according to Jewitt's order. In the maxmin model of ambiguity aversion with $u D C$, a marginal increase in wealth from $z$ raises the demand for the risky asset if relative prudence is uniformly smaller than relative risk aversion plus one.

To complete this section, let us consider the special case of HARA utility functions, i.e., functions with linear absolute risk tolerance:

$$
u(c)=\xi\left(\eta+\frac{c}{\gamma}\right)^{1-\gamma},
$$

for some scalars $\xi, \eta$, and $\gamma$. The consumption domain of this utility function is such that $\eta+c / \gamma$ is positive. We assume that $\xi(1-\gamma) / \gamma$ is positive to insure that $u$ is increasing and concave in its domain. DC holds if $\gamma$ is positive. The HARA set includes power, log, exponential and quadratic functions. Observe that

$$
\begin{gathered}
E u\left(z+\widetilde{x}_{i}\right)=\xi E\left(\hat{\eta}+\frac{\widetilde{x}_{i}}{\gamma}\right)^{1-\gamma}, \\
E u^{\prime}\left(z+\widetilde{x}_{i}\right)=\xi \frac{1-\gamma}{\gamma} E\left(\hat{\eta}+\frac{\widetilde{x}_{i}}{\gamma}\right)^{-\gamma},
\end{gathered}
$$

and

$$
E \widetilde{x}_{i} u^{\prime}\left(z+\widetilde{x}_{i}\right)=\xi \frac{1-\gamma}{\gamma} E \widetilde{x}_{i}\left(\hat{\eta}+\frac{\widetilde{x}_{i}}{\gamma}\right)^{-\gamma}
$$


where $\hat{\eta}=\eta+z / \gamma>0$. Observe that

$$
\begin{aligned}
E u\left(z+\widetilde{x}_{i}\right) & =\xi E\left(\hat{\eta}+\frac{\widetilde{x}_{i}}{\gamma}\right)\left(\hat{\eta}+\frac{\widetilde{x}_{i}}{\gamma}\right)^{-\gamma} \\
& =\xi \hat{\eta} E\left(\hat{\eta}+\frac{\widetilde{x}_{i}}{\gamma}\right)^{-\gamma}+\frac{\xi}{\gamma} E \widetilde{x}_{i}\left(\hat{\eta}+\frac{\widetilde{x}_{i}}{\gamma}\right)^{-\gamma} \\
& =\hat{\eta} \frac{\gamma}{1-\gamma} E u^{\prime}\left(z+\widetilde{x}_{i}\right)+(1-\gamma)^{-1} E \widetilde{x}_{i} u^{\prime}\left(z+\widetilde{x}_{i}\right)
\end{aligned}
$$

This implies that

$$
E u^{\prime}\left(z+\widetilde{x}_{i}\right)=\frac{1-\gamma}{\hat{\eta} \gamma} E u\left(z+\widetilde{x}_{i}\right)-\frac{1}{\hat{\eta} \gamma} E \widetilde{x}_{i} u^{\prime}\left(z+\widetilde{x}_{i}\right) .
$$

A symmetric condition holds for $\widetilde{x}_{j}$. This implies that

$$
\begin{aligned}
E u^{\prime}\left(z+\widetilde{x}_{i}\right)-E u^{\prime}\left(z+\widetilde{x}_{j}\right)= & \frac{1-\gamma}{\hat{\eta} \gamma}\left[E u\left(z+\widetilde{x}_{i}\right)-E u\left(z+\widetilde{x}_{j}\right)\right] \\
& +\frac{1}{\hat{\eta} \gamma}\left[E \widetilde{x}_{j} u^{\prime}\left(z+\widetilde{x}_{j}\right)-E \widetilde{x}_{i} u^{\prime}\left(z+\widetilde{x}_{i}\right)\right] .
\end{aligned}
$$

Suppose that the two left conditions in (14) hold. It implies that the first term in the RHS of the above equality vanishes, and that the second term is negative if $\gamma$ is positive, i.e., if $u$ is DC. This yields the following proposition.

Proposition 4 Suppose that $u$ is HARA and DC (condition (22) with $\gamma>$ $0)$. In the maxmin model of ambiguity aversion, an increase in wealth always increases the demand for the risky asset.

It is noteworthy that in the HARA subset with increasing concavity (condition (22) with $\gamma<0$ ) as the quadratic utility function, the demand for the risky asset is always decreasing in wealth.

\subsection{The KMM model}

Let's now consider the portfolio problem in the KMM smooth ambiguity framework. The decision problem becomes

$$
\alpha^{*}(z)=\operatorname{argmax}_{\alpha} E \phi\left(E u\left(z+\alpha \widetilde{x}_{\theta}\right)\right)
$$


We normalize again the unit of the risky asset in such a way that $\alpha^{*}(z)=1$. The first order condition is therefore

$$
E\left[\phi^{\prime}\left(E u\left(z+\widetilde{x}_{\widetilde{\theta}}\right)\right) E \widetilde{x}_{\widetilde{\theta}} u^{\prime}\left(z+\widetilde{x}_{\widetilde{\theta}}\right)\right]=0,
$$

We examine the condition under which the demand for the risky asset is increasing in wealth. Since the denominator in the relation

$$
\frac{d \alpha^{*}}{d z}=-\frac{E\left[\phi^{\prime \prime}\left(E u\left(z+\widetilde{x}_{\widetilde{\theta}}\right)\right) E u^{\prime}\left(z+\widetilde{x}_{\widetilde{\theta}}\right) E \widetilde{x}_{\widetilde{\theta}} u^{\prime}\left(z+\widetilde{x}_{\widetilde{\theta}}\right)\right]+E\left[\phi^{\prime}\left(E u\left(z+\widetilde{x}_{\widetilde{\theta}}\right)\right) E \widetilde{x}_{\widetilde{\theta}} u^{\prime \prime}\left(z+\widetilde{x}_{\widetilde{\theta}}\right)\right]}{E\left[\phi^{\prime \prime}\left(E u\left(z+\widetilde{x}_{\widetilde{\theta}}\right)\right)\left(E \widetilde{x}_{\widetilde{\theta}} u^{\prime}\left(z+\widetilde{x}_{\widetilde{\theta}}\right)\right)^{2}\right]+E\left[\phi^{\prime}\left(E u\left(z+\widetilde{x}_{\widetilde{\theta}}\right)\right) E \widetilde{x}_{\widetilde{\theta}}^{2} u^{\prime \prime}\left(z+\widetilde{x}_{\widetilde{\theta}}\right)\right]}
$$

is negative, this decreasing aversion property is satisfied if and only if

$$
E\left[\phi^{\prime \prime}\left(E u\left(z+\widetilde{x}_{\widetilde{\theta}}\right)\right) E u^{\prime}\left(z+\widetilde{x}_{\widetilde{\theta}}\right) E \widetilde{x}_{\widetilde{\theta}} u^{\prime}\left(z+\widetilde{x}_{\widetilde{\theta}}\right)\right]+E\left[\phi^{\prime}\left(E u\left(z+\widetilde{x}_{\widetilde{\theta}}\right)\right) E \widetilde{x}_{\widetilde{\theta}} u^{\prime \prime}\left(z+\widetilde{x}_{\widetilde{\theta}}\right)\right] \geq 0
$$

The second term in the left-hand side of this inequality is positive if $u$ is DC. Indeed, $u$ DC means that $A(c)=-u^{\prime \prime}(c) / u^{\prime}(c)$ is decreasing, which implies in turn that

$$
\begin{aligned}
E\left[\phi^{\prime}\left(E u\left(z+\widetilde{x}_{\widetilde{\theta}}\right)\right) E \widetilde{x}_{\widetilde{\theta}} u^{\prime \prime}\left(z+\widetilde{x}_{\widetilde{\theta}}\right)\right] & =-E\left[A\left(z+\widetilde{x}_{\widetilde{\theta}}\right) \phi^{\prime}\left(E u\left(z+\widetilde{x}_{\widetilde{\theta}}\right)\right) \widetilde{x}_{\widetilde{\theta}} u^{\prime}\left(z+\widetilde{x}_{\widetilde{\theta}}\right)\right] \\
& \geq-A(z) E\left[\phi^{\prime}\left(E u\left(z+\widetilde{x}_{\widetilde{\theta}}\right)\right) \widetilde{x}_{\widetilde{\theta}} u^{\prime}\left(z+\widetilde{x}_{\widetilde{\theta}}\right)\right] \\
& =-A(z) E\left[\phi^{\prime}\left(E u\left(z+\widetilde{x}_{\widetilde{\theta}}\right)\right) E \widetilde{x}_{\widetilde{\theta}} u^{\prime}\left(z+\widetilde{x}_{\widetilde{\theta}}\right)\right]=0 .
\end{aligned}
$$

We now turn to the analysis of the sign of the first term in (25). If we use the same method as for the second term, we can rewrite this first term as follows:

$$
\begin{aligned}
E\left[\phi^{\prime \prime}\left(E u\left(z+\widetilde{x}_{\widetilde{\theta}}\right)\right) E u^{\prime}\left(z+\widetilde{x}_{\widetilde{\theta}}\right) E \widetilde{x}_{\widetilde{\theta}} u^{\prime}\left(z+\widetilde{x}_{\widetilde{\theta}}\right)\right] & =-E\left[A_{\phi}\left(E u\left(z+\widetilde{x}_{\widetilde{\theta}}\right)\right) \phi^{\prime}\left(E u\left(z+\widetilde{x}_{\widetilde{\theta}}\right)\right) E u^{\prime}(z+\right. \\
& =-E\left[\xi(\widetilde{\theta}) \phi^{\prime}\left(E u\left(z+\widetilde{x}_{\widetilde{\theta}}\right)\right) E \widetilde{x}_{\widetilde{\theta}} u^{\prime}\left(z+\widetilde{x}_{\widetilde{\theta}}\right)\right]
\end{aligned}
$$

where $A_{\phi}(u)=-\phi^{\prime \prime}(u) / \phi^{\prime}(u)$ is the absolute measure of ambiguity aversion, and $\xi(\theta)=A_{\phi}\left(E u\left(z+\widetilde{x}_{\theta}\right)\right) E u^{\prime}\left(z+\widetilde{x}_{\theta}\right)$. Suppose that $\xi$ is a decreasing function of $\theta$, and that $E \widetilde{x}_{\theta} u^{\prime}\left(z+\widetilde{x}_{\theta}\right)$ satisfies the single-crossing property: there exists $\widehat{\theta}$ so that $(\theta-\widehat{\theta}) E \widetilde{x}_{\widetilde{\theta}} u^{\prime}\left(z+\widetilde{x}_{\widetilde{\theta}}\right) \geq 0$ for all $\theta$. From the above equality, this would imply that

$E\left[\phi^{\prime \prime}\left(E u\left(z+\widetilde{x}_{\widetilde{\theta}}\right)\right) E u^{\prime}\left(z+\widetilde{x}_{\widetilde{\theta}}\right) E \widetilde{x}_{\widetilde{\theta}} u^{\prime}\left(z+\widetilde{x}_{\widetilde{\theta}}\right)\right] \geq-\xi(\widehat{\theta}) E\left[\phi^{\prime}\left(E u\left(z+\widetilde{x}_{\widetilde{\theta}}\right)\right) E \widetilde{x}_{\widetilde{\theta}} u^{\prime}\left(z+\widetilde{x}_{\widetilde{\theta}}\right)\right]=0$.

This proves the following Lemma. 
Lemma 2 Suppose that $u$ is DC. In the portfolio problem under the KMM smooth ambiguity framework, the demand for the risky asset is increasing with wealth if $\xi(\theta)=A_{\phi}\left(E u\left(z+\widetilde{x}_{\theta}\right)\right) E u^{\prime}\left(z+\widetilde{x}_{\theta}\right)$ is decreasing in $\theta$ and there exists $\widehat{\theta}$ such that $(\theta-\widehat{\theta}) E \widetilde{x}_{\widetilde{\theta}} u^{\prime}\left(z+\widetilde{x}_{\widetilde{\theta}}\right) \geq 0$ for all $\theta$.

We can use this lemma to show that combining condition $\phi \mathrm{DC}$ to the one presented in Proposition 3 is sufficient for the demand for the risky asset to be increasing in wealth.

Proposition 5 Normalize the demand for the risky asset at wealth level $z$ to unity. Suppose that $E u\left(z+\widetilde{x}_{1}\right) \leq \ldots \leq E u\left(z+\widetilde{x}_{n}\right)$ and $\widetilde{x}_{1} \preceq_{J} \widetilde{x}_{2} \preceq_{J} \ldots \preceq_{J} \widetilde{x}_{n}$. In the KMM smooth ambiguity model, an increase in wealth around $z$ raises the demand for the risky asset if $u$ and $\phi$ are $D C$, and for all $x$ in the joint support of $\left\{\widetilde{x}_{1}, \ldots, \widetilde{x}_{n}\right\}$, condition (21) holds.

Proof: Because $u$ is DC, we have that $-u^{\prime}$ is more concave than $u$. Because $u$ prefers $\widetilde{x}_{\theta+1}$ to $\widetilde{x}_{\theta}, \widetilde{x}_{\theta+1} \succeq_{J} \widetilde{x}_{\theta}$ implies that the agent with utility function $-u^{\prime}$ also prefers $\widetilde{x}_{\theta+1}$ to $\widetilde{x}_{\theta}$, i.e., $E u^{\prime}\left(z+\widetilde{x}_{\theta+1}\right) \leq E u^{\prime}\left(z+\widetilde{x}_{\theta}\right)$. Because $A_{\phi}$ is decreasing, we obtain that $\xi(\theta)=A_{\phi}\left(E u\left(z+\widetilde{x}_{\theta}\right)\right) E u^{\prime}\left(z+\widetilde{x}_{\theta}\right)$ is decreasing in $\theta$. Moreover, condition (21) implies that $\psi(x)=x u^{\prime}(z+x)$ is more concave than $u$, so that $E \psi\left(\widetilde{x}_{\theta}\right)=E \widetilde{x}_{\theta} u^{\prime}\left(z+\widetilde{x}_{\theta}\right)$ is increasing in $\theta$. This is sufficient for the existence of an integer $\widehat{\theta}$ such that $(\theta-\widehat{\theta}) E \widetilde{x}_{\widetilde{\theta}} u^{\prime}\left(z+\widetilde{x}_{\widetilde{\theta}}\right) \geq 0$ for all $\theta$.Applying Lemma 2 yields the result.

A sufficient condition for $(21)$ is that $u$ has constant relative risk aversion.

Suppose that we replace the Jewitt's ordering condition by the stronger SSD condition: $\widetilde{x}_{1} \preceq_{S S D} \ldots \preceq_{S S D} \widetilde{x}_{n}$, or $E f\left(\widetilde{x}_{1}\right) \leq \ldots \leq E f\left(\widetilde{x}_{n}\right)$ for all $f$ increasing and concave. Because $u$ and $-u^{\prime}$ are increasing and concave, and assuming $A_{\phi}$ decreasing, we obtain that $\xi$ is monotone decreasing in $\theta$. The single crossing property for $E \widetilde{x}_{\theta} u^{\prime}\left(z+\widetilde{x}_{\theta}\right)$ requires another stochastic dominance order defined by Gollier (1995). Let us introduce define the location-weighted-probability function $T_{\theta}$ as follows:

$$
T_{\theta}(x)=\int^{x} t d F_{\theta}(t)
$$

where $F_{\theta}$ is the cumulative distribution function of $\widetilde{x}_{\theta}$. Following Gollier (1995), we say that $\widetilde{x}_{\theta+1}$ dominates $\widetilde{x}_{\theta}$ in the sense of Central Dominance (CD) if there exists a nonnegative scalar $m$ such that $T_{\theta}(x) \leq m T_{\theta+1}(x)$ for 
all $x$ in the joint support of $\widetilde{x}_{\theta}$ and $\widetilde{x}_{\theta+1}$. SSD-dominance is neither necessary nor sufficient for CD-dominance. Observe that any decreasing function $u^{\prime}$ can be expressed as a convex combinaison of decreasing step function: $\exists \sigma$ : $\mathbb{R} \rightarrow \mathbb{R}_{+}: \forall \in \rtimes \gg(\approx)$

$$
u^{\prime}(c)=\int \sigma(t) \operatorname{Ind}(c \leq t) d t
$$

This implies that

$$
E \widetilde{x}_{\theta} u^{\prime}\left(z+\widetilde{x}_{\theta}\right)=\int \sigma(t) T_{\theta}(t-z) d t
$$

If $\widetilde{x}_{\theta+1}$ dominates $\widetilde{x}_{\theta}$, then there exists $m \geq 0$ so that $E \widetilde{x}_{\theta} u^{\prime}\left(z+\widetilde{x}_{\theta}\right) \leq$ $m E \widetilde{x}_{\theta+1} u^{\prime}\left(z+\widetilde{x}_{\theta+1}\right)$. This implies that $\left[E \widetilde{x}_{\theta+1} u^{\prime}\left(z+\widetilde{x}_{\theta+1}\right) \leq 0 \Longrightarrow E \widetilde{x}_{\theta} u^{\prime}(z+\right.$ $\left.\left.\widetilde{x}_{\theta}\right) \leq 0\right]$, i.e., the single crossing property for $E \widetilde{x}_{\theta} u^{\prime}\left(z+\widetilde{x}_{\theta}\right)$. Using Lemma 2 , this proves the following Proposition.

Proposition 6 Suppose that $\widetilde{x}_{1} \preceq_{S S D} \quad \ldots \preceq_{S S D} \widetilde{x}_{n}$ and $\widetilde{x}_{1} \preceq_{C D} \quad \ldots \preceq_{C D}$ $\widetilde{x}_{n}$. In the KMM smooth ambiguity model, an increase in wealth raises the demand for the risky asset if $u$ and $\phi$ are $D C$.

This proposition is similar to the main result in Gollier (2011), who shows that an increase in ambiguity aversion reduces the demand for the risky asset in the KMM smooth ambiguity aversion framework if the priors can be ranked according to SSD and CD. The intersection of SSD and CD is not empty. For example, the well-known Monotone Likelihood Ratio (MLR) order is a subset of both SSD and CD.

Corollary 3 Suppose that $u$ and $\phi$ are $C D$. Then, the demand for the risky asset is decreasing in wealth if the set of priors can be ranked according to the $M L R$ order: $\forall\left(\theta, \theta^{\prime}\right) \in\{1, \ldots, n\}^{2}, d F_{\theta}(x) / d F_{\theta^{\prime}}(x)$ is monotone in $x$. 


\section{Conclusion}

Two basic hypotheses prevail in decision theory with a large consensus in the profession. The first one is that human being are averse to uncertainty. The second one is that they are decreasingly averse to uncertainty. In the classical expected utility, these properties of human behavior prevail respectively if the utility function $u$ is concave, and if it is decreasingly concave in the sense that $-u^{\prime \prime} / u^{\prime}$ is decreasing. In this paper, we have focused our attention to this concept of decreasing aversion, by examining two different decision problems when the decision maker is not ambiguity-neutral. We first define decreasing aversion by the property that the set of desirable uncertain prospects expands when wealth increases. In the smooth ambiguity aversion model, we have shown that the classical conditions of decreasing risk aversion and of decreasing ambiguity aversion imply this property, and that an intuitive weaker condition is necessary and sufficient. In the $\alpha$-MEU model, the standard DARA condition is necessary and sufficient.

Another definition of decreasing aversion is that the demand for a risky asset is increasing with wealth. The introduction of the ingredient of ambiguitysensitive preferences into the picture implies much more complexity than in the above discrete choice problem. Even in the simpler maxmin criterion, the decreasing concavity of $u$ is not sufficient to get this result, except in the case of small risks. As in the smooth ambiguity aversion model with a decreasingly concave ambiguity-related function $\phi$, the unambiguous comparative static result requires some assumptions on the structure of ambiguity. As in Jewitt and Mukerji (2011), this paper illustrates once again the fact that even the most intuitive departures from the classical subjective expected utility model introduce much richness to our decision models. This is at the cost of a non-marginal increment in the complexity of the analysis.

\section{References}

Arrow, K.J., (1963), Liquidity preference, Lecture VI in "Lecture Notes for Economics 285, The Economics of Uncertainty", pp 33-53, undated, Stanford University.

Dow, J., and S. Werlang, (1992), Uncertainty aversion, risk aversion and the optimal choice of portfolio, Econometrica, 60, 197-204. 
Ellsberg, D., (1961), Risk, ambiguity, and the Savage axioms, Quarterly Journal of Economics, 75, 643-69.

Gilboa, I. and D. Schmeidler, (1989), Maximin expected utility with non-unique prior, Journal of Mathematical Economics, 18, 141, 153.

Ghirardato, P., F. Maccheroni, and M. Marinacci, (2004), Differentiating ambiguity and ambiguity attitude, Journal of Economic Theory, 118, 133-173.

Gollier, C., (1995), "The Comparative Statics of Changes in Risk Revisited", Journal of Economic Theory 66, 522-535.

Gollier, C., (2001), The economics of risk and time, MIT Press, Cambridge.

Jewitt, I., (1989), Choosing between risky prospects: the characterization of comparative statics results, and location independent risk, Management Science, 35, 60-70.

Jewitt, I., and S. Mukerji, (2011), Ordering ambiguous acts, unpublished manuscript, Department of Economics, Oxford University.

Kimball, M.S., (1990), Precautionary savings in the small and in the large, Econometrica, 58, 53-73.

Klibanoff, P., M. Marinacci and S. Mukerji, (2005), A smooth model of decision making under ambiguity, Econometrica 73(6), 1849-1892.

Rothschild, M. and J. Stiglitz, (1970), Increasing Risk: I. A Definition, Journal of Economic Theory, 2, 225-243.

Rothschild, M. and Stiglitz, J.E., (1971), Increasing Risk II: Its Economic Consequences, Journal of Economic Theory, 3, pp. 225-243. 


\section{Appendix: The wealth effect in the maxmax portfolio problem}

In this Appendix, we present a short analysis of the portfolio choice problem when the investor has a maxmax preference functional:

$$
\max _{\alpha} \max _{\theta} E u\left(z+\alpha \widetilde{x}_{\theta}\right) .
$$

In the family of $\alpha-\mathrm{MEU}$ preferences, this model is the polar one to the maxmin criterion. Of course, $u \mathrm{DC}$ is necessary to guarantee that $\alpha$ is increasing in $z$ in intervals of wealth levels where the argument of the maximum of $E u\left(z+\alpha \widetilde{x}_{\theta}\right)$ with respect to $\theta$ does not change. Because the objective function is not concave with respect to the decision variable $\alpha$, we also need to take care of the possible bifurcations. In Figure 3, we describe a situation in which the demand for the risky asset goes discountinuously down from $\alpha(z)$ to $\alpha\left(z^{\prime}\right)<\alpha(z)$ when wealth goes up from $z$ to $z^{\prime}$.

We first show that bifurcations never occur in the case of HARA utility functions defined by (22). Let us define

$$
V_{\theta}(z)=\max _{\alpha} E u\left(z+\alpha \widetilde{x}_{\theta}\right)
$$

The absence of bifurcation comes from the property that, for all $(i, j) \in$ $\{1, \ldots, n\}^{2}$,

$$
V_{i}(z) \geq V_{j}(z) \Longrightarrow \forall z^{\prime}>-\eta \gamma: \quad V_{i}\left(z^{\prime}\right) \geq V_{j}\left(z^{\prime}\right) .
$$

In other words, the $\theta$ that maximizes expected utility with the optimal portfolio never switches in the case of HARA preferences (22). To show this, let us observe that conditional to $\theta$, the expected-utility-maximizing investment in the risky asset is linear in the wealth level $z$. The first-order condition to the maximization of $E u\left(z+\alpha \widetilde{x}_{\theta}\right)$ can be written as follows:

$$
E \widetilde{x}_{\theta}\left(\eta \gamma+z+\alpha_{\theta}^{*}(z) \widetilde{x}_{\theta}\right)^{-\gamma}=0 .
$$

Let us define $\alpha_{\theta}^{*}$ as the unique root of the follwoing equation:

$$
E \widetilde{x}_{\theta}\left(1+\alpha_{\theta}^{*} \widetilde{x}_{\theta}\right)^{-\gamma}=0 .
$$




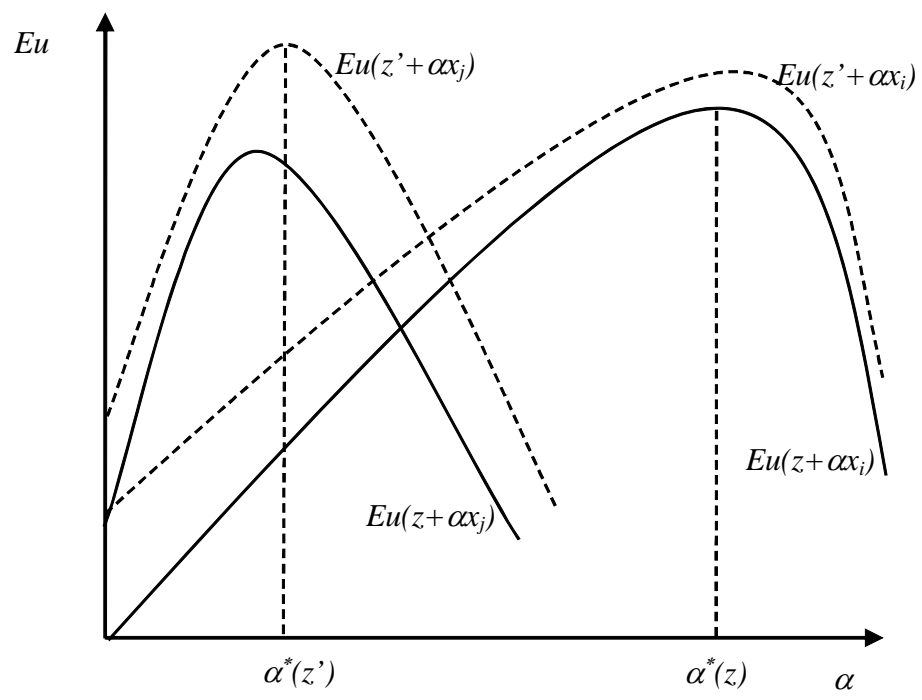

Figure 3: A bifurcation yielding a decreasing demand for the risky asset in the maxmax portfolio model. 
By comparing the last two equations, it is immediate that the optimal solution conditional to $\theta$ is $\alpha_{\theta}^{*}(z)=\alpha_{\theta}^{*}(\eta \gamma+z)$ for all $z>-\eta \gamma$. We can now compute $V_{\theta}(z)$. We obtain:

$$
V_{\theta}(z)=\xi E\left(\frac{(\eta \gamma+z)\left(1+\alpha_{\theta}^{*} \widetilde{x}_{\theta}\right)}{\gamma}\right)^{1-\gamma}=(\eta \gamma+z)^{1-\gamma} v_{\theta}^{*}
$$

with

$$
v_{\theta}^{*}=\xi E\left(\frac{1+\alpha_{\theta}^{*} \widetilde{x}_{\theta}}{\gamma}\right)^{1-\gamma} .
$$

It implies that the $\left(V_{1}(z), \ldots, V_{n}(z)\right)$ can be ordered in the same way as $\left(v_{1}^{*}, \ldots, v_{n}^{*}\right)$, which is independent of $z$. This implies in particular that the largest element in $\left(V_{1}(z), \ldots, V_{n}(z)\right)$ is independent of $z$. Hence, there is no bifurcation. This concludes the proof of the following result.

Proposition 7 Consider the maxmax portfolio problem (27) with a HARA utility function $u$. In this framework, there is no bifurcation when wealth increases in the sense that the $\theta$ that maximizes the expected utility along the optimal portfolio strategy is independent of $z$. This implies that $u D C$ is sufficient to guarantee that the demand for the risky asset is increasing with wealth in the maxmax-HARA portfolio model.

One can easily find a counterexample where the bifurcation yields a downward jump in the demand for the risky asset when wealth increases in spite of DARA. Consider the DARA utility function $u(c)=c-k_{1} e^{-k_{2} c}$ with $k_{1}=10$ and $k_{2}=1$. Consider an ambiguous situation with two possible priors: $\widetilde{x}_{1} \sim(-1,1 / 2 ; 2,1 / 2)$ and $\widetilde{x}_{2} \sim(-1,1 / 3 ; 1,2 / 3)$. The demand for the risky asset as a function of wealth is represented in Figure 4. This illustrates the fact that DARA is not sufficient for a monotone relationship between wealth and the optimal exposure to risk in the maxmax model.

Let us now characterize bifurcations. A bifurcation occurs at some wealth level $z$ if two global maxima to problem (27) prevails for that $z$. Let $\widetilde{y}_{\theta}$ denote $\alpha_{\theta}^{*} \widetilde{x}_{\theta}$. The following set of conditions is necessary for $\left(\alpha_{i}^{*}, \alpha_{j}^{*}\right)$ to be two global maxima:

$$
\begin{aligned}
& E \widetilde{y}_{i} u^{\prime}\left(z+\widetilde{y}_{i}\right)=0, \\
& E \widetilde{y}_{j} u^{\prime}\left(z+\widetilde{y}_{j}\right)=0,
\end{aligned}
$$




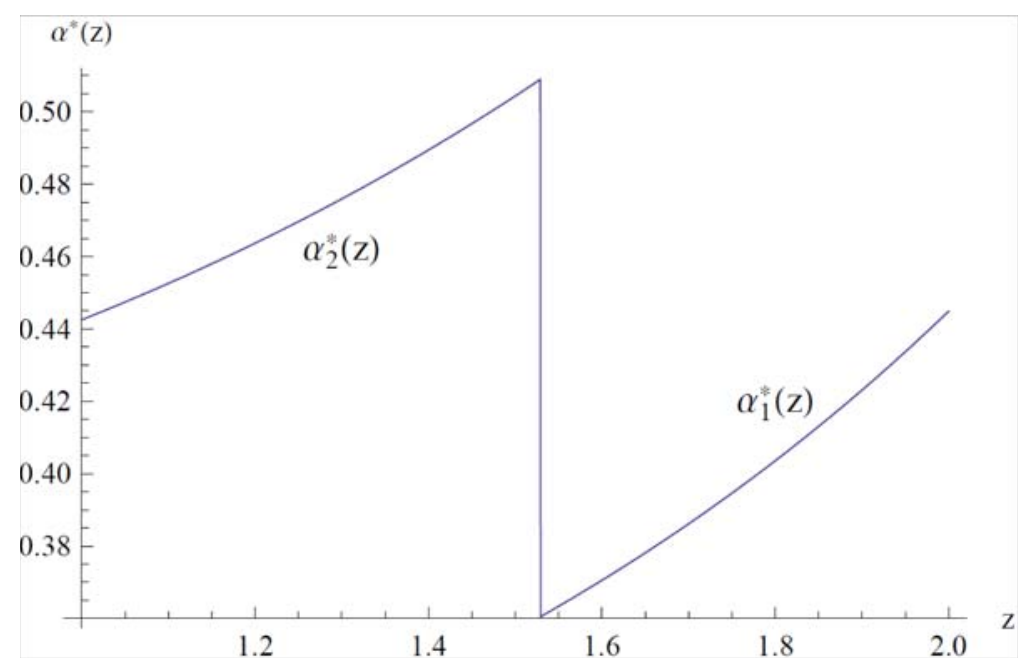

Figure 4: The demand for the risky asset as a function of wealth in the maxmax model.

and

$$
E u\left(z+\widetilde{y}_{i}\right)=E u\left(z+\widetilde{y}_{j}\right) .
$$

A marginal increase in wealth yields a bifurcation from $\alpha_{j}^{*}$ to $\alpha_{i}^{*}$ iff

$$
E u^{\prime}\left(z+\widetilde{y}_{i}\right)>E u^{\prime}\left(z+\widetilde{y}_{j}\right) .
$$

Whether this bifurcation is demand-increasing or demand-decreasing depends upon whether $\alpha_{i}^{*} \geq \alpha_{j}^{*}$ or $\alpha_{i}^{*} \leq \alpha_{j}^{*}$, respectively. If it is a demandincreasing bifurcation, we can build another ambiguous context with the same utility function in which there is a demand-decreasing bifurcation.

Proposition 8 Consider the maxmax model with utility function $u$. Consider an ambiguous context $\left(\widetilde{x}_{1}, \ldots, \widetilde{x}_{n}\right)$ with $E \widetilde{x}_{\theta}>0$ for all $\theta=1, \ldots, n$ such that there is a demand-increasing bifurcation at wealth level $z$. Then, there exists another ambiguous context $\left(\widetilde{x}_{1}^{\prime}, \ldots, \widetilde{x}_{n}^{\prime}\right)$ such that there is a demanddecreasing bifurcation at wealth $z$.

Proof: Observe first that condition $E \widetilde{x}_{\theta}>0$ implies that the local maximum $\alpha_{\theta}^{*}$ is positive. Suppose that the two local maxima under $\left(\widetilde{x}_{1}, \ldots, \widetilde{x}_{n}\right)$ 
are $\alpha_{i}^{*}$ and $\alpha_{j}^{*}$, with $\alpha_{i}^{*}>\alpha_{j}^{*}$. It implies that $\widetilde{y}_{i}=\alpha_{i}^{*} \widetilde{x}_{i}$ and $\widetilde{y}_{j}=\alpha_{j}^{*} \widetilde{x}_{j}$ satisfy conditions (28)-(31), yielding a demand-increasing bifurcation at $z$. Consider now the alternative ambiguous context $\left(\widetilde{x}_{1}^{\prime}, \ldots, \widetilde{x}_{n}^{\prime}\right)$ such that $\widetilde{x}_{\theta}^{\prime}=\widetilde{x}_{\theta}$ for all $\theta \neq i$ and $\widetilde{x}_{i}^{\prime}=k \widetilde{x}_{i}$ where $k$ is a positive scalar larger than $\alpha_{i}^{*} / \alpha_{j}^{*}>0$. It is then immediate that the pair $\left(\alpha_{i}^{* \prime}=\alpha_{i}^{*} / k, \alpha_{j}^{*}\right)$ describes two global maxima under the new ambiguous context. Indeed, we have that $\alpha_{i}^{*} \widetilde{x}_{i}^{\prime}=\alpha_{i}^{*} \widetilde{x}_{i}=\widetilde{y}_{i}$, so that the pair of conditions (28)-(31) is preserved by the joint change of ambiguity context and in $\left(\alpha_{i}^{*}, \alpha_{j}^{*}\right)$. But in the new context, we have that

$$
\alpha_{i}^{* \prime}=\frac{\alpha_{i}^{*}}{k}<\frac{\alpha_{i}^{*} \alpha_{j}^{*}}{\alpha_{i}^{*}}=\alpha_{j}^{*}
$$

so that the bifurcation is now demand-decreasing.

This proposition tells us that as soon as an ambiguity context yields a bifurcation in the demand for the risky asset as some wealth level, we can make it demand-decreasing, thereby violating the desired comparative statics property. We have seen earlier that there is never any bifurcation if $u$ is HARA. In the remainder of this section, we examine the case of small risks.

Lemma 3 Suppose that there are two local maxima $\alpha_{i}^{*}$ and $\alpha_{j}^{*}$ to program (27) at wealth level $z$, and that they are small. This requires that

$$
m_{1 \theta}-\alpha_{\theta}^{*} m_{2 \theta} A+0.5 \alpha_{\theta}^{* 2} m_{3 \theta} P=0
$$

for $\theta=i$ and $j$, together with

$$
\alpha_{i}^{* 2} m_{2 i}-\alpha_{j}^{* 2} m_{2 j}=\frac{2}{3}\left[\alpha_{i}^{* 3} m_{3 i}-\alpha_{j}^{* 3} m_{3 j}\right] P,
$$

where $m_{k \theta}$ is the kth moment of $\widetilde{x}_{\theta}$ and $A$ and $P$ are respectively the absolute risk aversion and the absolute prudence evaluated at wealth level z. A marginal increase in $z$ implies a bifurcation from $\alpha_{j}^{*}$ to $\alpha_{i}^{*}$ if and only if

$$
\left[\alpha_{i}^{* 2} m_{2 i}-\alpha_{j}^{* 2} m_{2 j}\right][A+T-2 P] \leq 0,
$$

where $T=-u^{\prime \prime \prime \prime}(z) / u^{\prime \prime \prime}(z)$ is the index of absolute temperance. 
Proof: Condition (32) is obtained from (28) and (29) via second-order Taylor expansion $u^{\prime}(z+\alpha x)$ around $z$. Using third-degree Taylor expansions, condition (30) can be rewritten as follows:

$$
\alpha_{i}^{*} m_{1 i}-\frac{1}{2} \alpha_{i}^{* 2} m_{2 i} A+\frac{1}{6} \alpha_{i}^{* 3} m_{3 i} A P=\alpha_{j}^{*} m_{1 j}-\frac{1}{2} \alpha_{j}^{* 2} m_{2 j} A+\frac{1}{6} \alpha_{j}^{* 3} m_{3 j} A P .
$$

Replacing $m_{1 i}$ and $m_{1 j}$ in this equation by their expression derived from (32) yields condition (33). Using the same method for inequality (31) yields

$$
\left[\alpha_{i}^{* 2} m_{2 i}-\alpha_{j}^{* 2} m_{2 j}\right]\left[A-\frac{1}{2} P\right] \leq\left[\alpha_{i}^{* 3} m_{3 i}-\alpha_{j}^{* 3} m_{3 j}\right]\left[\frac{A}{2}-\frac{T}{6}\right] P .
$$

Using condition (33) to eliminate $\alpha_{i}^{* 3} m_{3 i}-\alpha_{j}^{* 3} m_{3 j}$ in this inequality yields condition (34).

Observe that in the HARA case, $A+T-2 P$ is uniformly zero, so that if there are two global maxima to program (27) for some $z$, this is the case for all $z$. With a non-HARA utility function, whether bifurcations are compatible with an increasing demand for the risky asset depends upon a complex condition linking the signs of $A+T-2 P$ and of $\alpha_{i}^{* 2} m_{2 i}-\alpha_{j}^{* 2} m_{2 j}$, where $\alpha_{i}^{*}$ and $\alpha_{j}^{*}$ are defined by (32) under constraint (33). Notice that skewness is important. On the contrary, if $\widetilde{x}_{i}$ and $\widetilde{x}_{j}$ are such that $m_{3 i}=m_{3 j}=0$ implies that $m_{2 i}=m_{2 j}$ by condition (33) to guarantee that the two local maxima are also global maxima. But this latter condition implies that condition (34) is satisfied as an equality, which means that there is no bifurcation. 